\title{
Kikuchi's Disease Presenting as Pyrexia of Unknown Origin: A Case Report \& Literature Review
}

\author{
Authors \\ Dr Priya Sahu ${ }^{1}$, Dr Ashish Nigam ${ }^{2}$ \\ Max Super Speciality Hospital, Saket, New Delhi, India \\ Corresponding Author \\ Dr Priya Sahu \\ K-40/D, Sheikh Sarai Phase II, New Delhi. 110017 \\ Email:drpriyanigam@gmail.com.Mobile Number-9560556590
}

\begin{abstract}
We report this case of a 41 year old Indian male who presented as pyrexia of unknown origin along with weight loss and joint pain. The patient underwent extensive laboratory investigations with clinical suspicion of tubercular or viral etiologies; except for elevated ESR and mildly low leukocyte count all the laboratory investigations were normal. Radiological examinations showed generalized lymphadenopathy and hepatosplenomegaly. The final diagnosis was reached by histopathological examination of cervical lymph node, which showed necrotic foci with karyorrhectic and eosinophilic debris surrounded by lymphohistiocytic and mononuclear plasmacytoid cells which are characteristics of Kikuchi-Fujimoto disease (KFD), so-called histiocytic Necrotizing lymphadenitis.
\end{abstract}

Keywords: Kikuchi-Fujimoto disease, Histiocytic necrotizing lymphadenitis, Lymphadenopathy, Pyrexia of unknown origin.

\section{Introduction}

Kikuchi disease is an uncommon, benign and rarely fatal disease. It affects primarily the cervical lymph nodes of young adults strikingly prevalent in females and has a self-limited clinical course. ${ }^{[1]}$ The usual presentation is lymphadenopathy with tenderness, mild fever and night sweats. Less frequent symptoms include weight loss, nausea, vomiting and sore throat. The pathogenesis is poorly understood, but is thought to be a hyperimmune response induced by different antigenic stimuli or an autoimmune process in which apoptosis plays an important role. ${ }^{[2]}$

\section{Case Report}

A 41 years old male presented with complaints of weight loss, fever and joint pain for three weeks. History of recent travel was there; where he started fever and received an antibiotics course. Clinical examination revealed $102 \mathrm{~F}$ temperature, left cervical swelling and tenderness over the bilateral knee, ankle and wrist joints. The rest of the clinical examinations were unremarkable. There was no past history of any medical illness or drug allergy.

Various hematological, serological and biochemical tests were performed and except of raised ESR and mildly low leukocyte count, all were within normal limits. Chest X-ray revealed prominent bilateral 
hilar shadows. Ultrasound of the whole abdomen showed hepatosplenomegaly. CT chest, abdomen and pelvis demonstrated mediastinal, bilateral hilar, left cervical, left supraclavicular and left axillary lymphadenopathy with hepatosplenomegaly. PET CT was done in view of pyrexia of unknown origin which showed FDG avid and non avid cervical, mediastinal, axillary and abdominal lymph nodes. (Fig.1) No other significant FDG avid lesion in the body surveyed. Surgical reference was taken for cervical lymph node biopsy. Microscopic examination showed discrete areas of necrosis with incomplete architectural effacement. (Fig.2) Karyorrhectic nuclear debris with associated mononuclear cell response composed of histiocytes, lymphoid cells and plasmacytoid cells with no granulocytic infiltration was seen.(Fig.3,4) Workup for tuberculosis was negative. Final diagnosis was arrived as Kikuchi's disease.

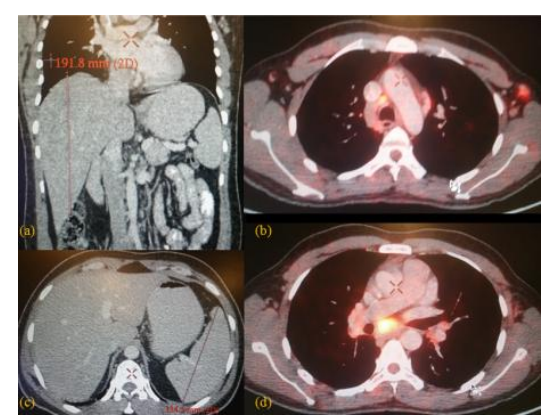

Figure 1 Coronal (a) and axial (c) contrast enhanced CT of abdomen demonstrate hepatosplenomegaly; Axial (b) \& (d) PET CT demonstrate FDG avid and non avid left axillary, mediastinal and bilateral hilar lymph nodes.

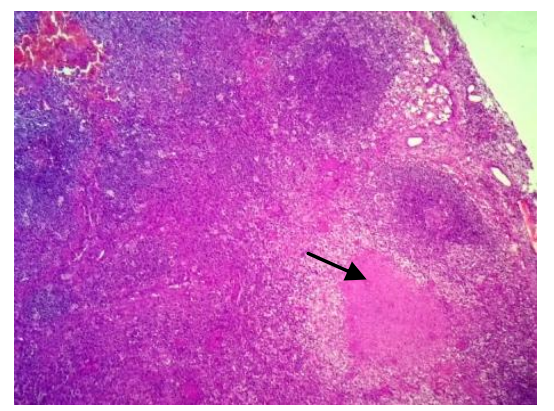

Figure 2 Lymph node biopsy shows necrotic foci, $\mathrm{H} \& \mathrm{E}, 4 \mathrm{x}$ view.

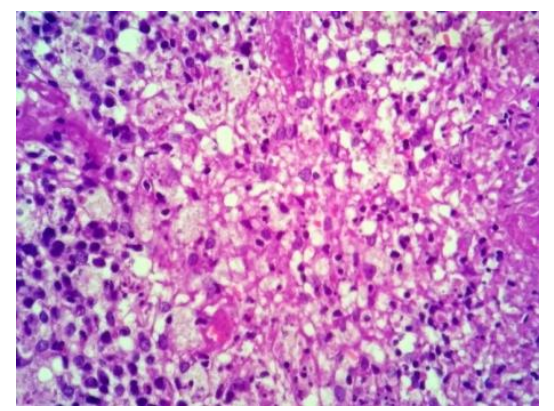

Figure 3 Section shows mononuclear cell infiltrates adjacent to necrotic foci, H\&E, 40x view

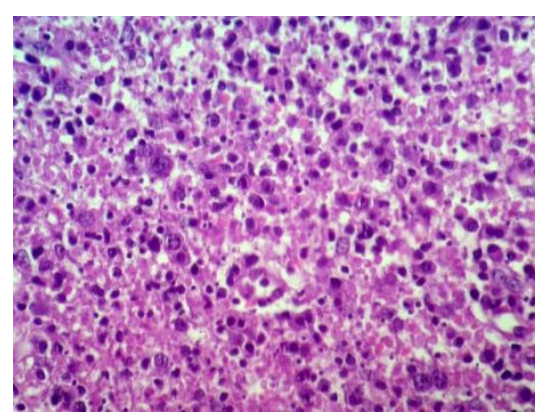

Figure 4 Section shows karyorrhectic nuclear debris and phagocytic histiocytes, H\&E, 40x view

\section{Discussion}

The combination of pyrexia of unknown origin, weight loss and lymphadenopathy particularly in Indian scenario often leads to an initial, incorrect diagnosis of an infectious process such as tuberculosis or viral etiologies; so as in this case.

Histiocytic necrotizing lymphadenitis or KikuchiFujimoto disease (KFD) was first described independently in 1972 by Kikuchi ${ }^{[3]}$ and Fujimoto et al ${ }^{[4]}$ in Japan. Its incidence has been reported worldwide with a higher prevalence among Japanese and other Asian descendents.

KFD usually involve cervical lymph nodes; however, involvement of axillary, mesenteric, mediastinal, inguinal, intra parotid, iliac, celiac and peripancreatic lymph nodes has been reported as well. ${ }^{[5][6][7]}$ KFD is thought to have three evolving phases: proliferative, necrotizing and xanthomatous. No specific laboratory tests contribute to the diagnosis. Histopathologic examination is required to make an accurate diagnosis. Exclusion of other factors can be done by ancillary studies.

It is very important to differentiate this entity among necrotizing lymphadenitis and number of infectious, autoimmune and lymphoproliferative lymphadeno- 
pathies, as they all have overlapping clinicopathological features and different treatment protocols as well as prognoses.

The distinction from tubercular lymphadenitis is crucial, especially in Indian context, where tuberculosis is rather common. The epithelioid cell granulomas, multi-nucleated Langhans giant cells, caseous necrosis and absence of karyorrhectic debris clearly favor the diagnosis of tuberculosis.

Systemic lupus erythematosus (SLE) is most challenging differential diagnosis, characterized by presence of numerous plasma cells, hematoxyphilic bodies (aggregates of degenerative nuclear debris) and neutrophilic infiltration favor a diagnosis of SLE, while absence of granulocytic infiltration favors KFD.

Malignant lymphoma should also be ruled out owing to the overlapped clinical and histologic features. Immunohistochemistry is also an important tool in distinguishing KFD from high grade lymphomas. Histologically, the lymph nodes of patients with KFD show mixed cellular proliferation consisting of lymphoid blast cells of varying size (so-called plasmacytoid cells), which are believed to be of monocyte lineage. Immunohistochemistry shows a positive immunostaining by antibodies Mac 387, KP1 (CD 68) and Ki M1P in KFD. In addition, a variable number of $\mathrm{T}$ cells immunostained by antibodies MT1 (CD 43) or UCHL1 and (CD 45 RO). ${ }^{[8]}$

The clinical course of KFD is self-limiting, the symptoms usually resolve spontaneously within one to six months of initial onset. However, low recurrence rate $(3 \% \text { to } 4 \%)^{[1]}$ and rare fatal cases have been documented in the literature. ${ }^{[9]}$ In general, treatment involves supportive measures that include relief from fever, joint pain and lymph node tenderness with use of antipyretics and analgesics. Corticosteroids are reserved for severe cases or relapsing disease. Because of association with SLE, it is suggested that patient should receive a systemic survey and follow-up to evaluate subsequent development of SLE. ${ }^{[10]}$

\section{Conclusions}

Awareness of this uncommon condition amongst Clinician's and pathologists is decisive to prevent misdiagnosis and inappropriate treatment. KFD is a self limiting disease and responds well to symptommatic treatment. Early and accurate diagnosis can curtail the need of lengthy and costly diagnostic investigations and therapeutic interventions.

\section{References}

1. Kuo T. Kikuchi's disease (histiocytic necrotising lymphadenitis): a clinicopathologic study of 79 cases with an analysis of histologic subtypes, immunohistology and DNA ploidy. Am J Surg Pathol 1995;19:798-809.

2. Menasce LP, Banerjee SS, Edmondson D, Harris M. Histiocytic necrotizing lymphadenitis (Kikuchi-Fujimoto disease): continuing diagnostic difficulties. Histopathology. 1998; 33 (3): 248-54

3. Kikuchi M. Lymphadenitis showing focal reticulum cell hyperplasia with nuclear debris and phagocytes. Acta Hematol Jpn 1972; 35:379-80.

4. Fujimoto Y, Kozima Y, Yamaguchi K. Cervical subacute necrotizing lymphadenitis.

A new clinicopathological entity. Naika 1972; 30:920-7

5. Thongsuksai P, Kayasut K. Histiocytic necrotizing lymphadenitis (Kikuchi's disease): clinicopathologic characteristics of 23 cases and literature review. J Med Assoc Thai. 1999;82:812-818.

6. Madle-Samardzija N, Turkulov V, Vukadinov J, Stajnic S, Canak G. Histiocytic necrotizing lymphadenitis (Kikuchi-Fujimoto disease). Med Pregl. 2000;53: 513-516.

7. Yabe H, Sinzato I, Hashimoto K. Necrotizing lymphadenitis presenting as mesenteric lymphadenopathy. Rinsho Ketsueki. 1999;40:658-662.

8. Hassan M, Anees A, Zaheer S. KikuchiFujimoto disease: diagnostic dilemma and 
the role of immunohistochemistry. J Clin Med Res. 2009;1(4):244-246.

9. Kucukardali Y, Solmazgul E, Kunter E, Oncul O, Yildirim S, Kaplan M. KikuchiFujimoto Disease: analysis of 244 cases. Clin Rheumatol. 2007;26(1):50-4. doi: 10.1007/s10067-006-0230-5.

10. Ramkumar A. Kikuchi-Fujimoto Disease as a Differential Diagnosis for Cervical Lymphadenopathy in India: A Case Report and Review of Literature. Indian Journal of Otolaryngology and Head \& Neck Surgery. 2011;63(Suppl 1):110-112. 